BMJ Open Sport \& Exercise Medicine

\title{
Pilot evaluation of risk assessment and enhanced protocols regarding contacts at an international professional golf event
}

\author{
Patrick G Robinson (D , , ${ }^{1,2}$ Andrew Murray, ${ }^{2,3,4}$ Volker Sheer, ${ }^{2,5,6}$ Graeme Close, ${ }^{2,7}$ \\ Denis F Kinane ${ }^{8,9}$
}

To cite: Robinson PG, Murray A, Sheer V, et al. Pilot evaluation of risk assessment and enhanced protocols regarding contacts at an international professional golf event. $B M J$ Open Sport \& Exercise Medicine 2021;7:e001127. doi:10.1136/ bmjsem-2021-001127

Accepted 2 August 2021

Check for updates

C Author(s) (or their employer(s)) 2021. Re-use permitted under CC BY-NC. No commercial re-use. See rights and permissions. Published by BMJ.

${ }^{1}$ Edinburgh Orthopaedics, Royal Infirmary of Edinburgh, Edinburgh, UK

${ }^{2}$ European Tour Performance Institute, Virginia Water, UK ${ }^{3}$ Physical Activity for Health Research Centre, University of Edinburgh, Edinburgh, UK ${ }^{4}$ Sport and Exercise, University of Edinburgh, Edinburgh, UK ${ }^{5}$ Health Science Department, Universidad a Distancia de Madrid (UDIMA), Madrid, Spain ${ }^{6}$ Ultra Sports Science

Foundation, Pierre Benite, France

Research Institute for Sport and Exercise Sciences, Liverpool John Moores University, Liverpool, UK

${ }^{8}$ School of Dental Medicine, University of Bern, Bern Switzerland

${ }^{9}$ Medical and Scientific Department, Cignpost Diagnostics, Farnborough, UK

Correspondence to Dr Patrick G Robinson; patrick.robinson321@gmail.com

\section{ABSTRACT}

Objectives The aim of this study was to assess whether a risk assessment and managed risk approach to contact tracing was practical and feasible at the Gran Canaria Lopesan Open 2021 and could inform further pilot work regarding disease transmission during elite sporting events.

Methods This prospective cohort study included all international attendees. All participants required a minimum of one negative reverse transcriptase PCR (RT-PCR) test prior to travelling to each tournament. Highrisk contacts were isolated for 10 days. Moderate-risk contacts received education regarding enhanced medical surveillance, had daily rapid antigen testing for 5 days, with RT-PCR day 5, mandated mask use and access to outside space for work purposes only. Low-risk contacts received rapid antigen testing every 48 hours and PCR testing on day 5 .

Results A total of 550 persons were accredited and were required to undergo RT-PCR testing before the event. Two of these tests were positive $(0.36 \%)$. Of these, case 1 had 1 high, 23 moderate and 48 low-risk contacts. Case 2 did not have any significant travel history within 2 days of positive test and had one high-risk contact. There were no further positive tests on site in the wider cohort of attendees, from a total of 872 RT-PCR and 198 rapid antigen tests.

Conclusions This pilot study showed it is practical, feasible and well accepted to provide enhanced (daily) virus testing and risk-mitigating measures at a professional golf event. Further study is required to assess the efficacy of these interventions; however, no transmission was found in this pilot study.

\section{INTRODUCTION}

The WHO have been consistent in their support and encouragement for the physical and mental health benefits that physical activity and sport can provide. ${ }^{1}$ In the face of a pandemic, this has been supported by evidence regarding the benefits of physical activity for immune function. ${ }^{2}$ Elite and professional sport can provide economic, social and wider benefits. However, such

\section{Key messages}

What is already known

- International policies have been developed to protect public health and facilitate elite sporting events.

- Disease transmission is very low in outdoor sporting environments.

- A risk-based model for contact tracing is being piloted in various workplace settings aiming to maintain low transmission while minimising unnecessary episodes of self-isolation.

What are the new findings

- Protocols for minimised risk environments and enhanced testing can be well tolerated by professional golfers and their essential support staff.

- Daily testing is both practical and feasible and may provide an alternative to self-isolation in the correct context.

- This approach merits further evaluation to assess whether it can protect participation in golf and other sports, while not increasing risk of disease transmission

events can only be conducted with risk assessment and appropriate measures regarding transmission of COVID-19.

Assessing the risk of disease contraction and transmission is a dynamic process, with consideration required of (1) local incidence of COVID-19, (2) vaccination rates and (3) the infrastructure to support sporting events. The WHO has provided key recommendations regarding mass gatherings, ${ }^{3}$ event organisation $^{4}$ and risk assessment specific to sport. ${ }^{5}$ These documents can continue to serve as a foundational framework, with more detailed guidance appropriate to a specific country $^{6} 7$ by governing sporting bodies. In addition to 'the science', decisions regarding sporting events are also be informed by wider societal and political factors.

Elite and professional sports have put in place risk assessment and control measures, 
which have been shown to be effective in returning risk to, or below baseline population levels, even with international travel. ${ }^{8-10}$ Collaborative work has seen a return in most countries to training and competition, and in some, a return to cross-border competition and/ or spectator attendance. Contact tracing, along with robust testing, isolation and care of cases, is a key strategy for interrupting chains of transmission of SARS-CoV-2 and reducing COVID-19-associated mortality. ${ }^{11}$ Given an increased knowledge of factors related to transmission, ${ }^{12}$ and the enhanced medical and other resources available to elite sport, there may be instances where individuals who meet criteria as a close contact could participate, if the risk of onward transmission to the public is very low. This would only be appropriated with approval from the event medical director and host public health authority.

The track and trace system implemented globally has been effective in curbing the spread of COVID-19; ${ }^{13-15}$ however, its blanket application, regardless of risk, has had significant disruption on society including the conduct of elite sporting events. Nuanced approaches based on individual risk have been proposed by public health authorities and experts in public health. ${ }^{16}$ One research group from the USA has designed and proven the efficacy of a 'risk-based quarantine' model, developing contact tracing policies that are effective in reducing disease transmission while requiring less quarantine. $^{17}$

Following conversations with the International Chief Medical Officer Group for Sport, the WHO Mass Gatherings team and host public health teams, a risk assessment and managed risk approach were piloted for professional golf at the Gran Canaria Lopesan Open 17-25 April 2021. The aim of this study was to assess whether a risk-assessed approach was practical to undertake at a major sporting event while also enabling a safe environment for those participating. Specifically, we aim to establish whether using a risk-assessed approach to guide testing and isolation of event attendees who were COVID-19 contacts can be performed safely and subsequently inform further research regarding disease transmission during elite sporting events

\section{METHODS}

This prospective, cohort study included all national and international attendees of the Gran Canaria Lopesan Open. The study period was commenced on 17 April 2021 and concluded on 28 April 2021. Players attended from 27 countries of which $84 \%$ were from European countries. All participants required a minimum of one negative reverse transcriptase PCR (RT-PCR) test prior to travelling to each tournament, except those attending within 90 days of a confirmed positive RT-PCR test. ${ }^{18}$ All event attendees were also required to retest on site. This was performed using a nasopharyngeal and oropharyngeal swab taken by a trained professional. Testing was conducted by Cignpost Diagnostics for RT-PCR on the MicoBioMed (MicoBioMed, Seoul, South Korea) or
Table 1 Player questionnaire completed before attending venue each day

Question

Yes/no

1. Any new continuous cough?

2. Any new shortness of breath?

3. Any new fever (ie, feeling hot or cold to touch)?

4. Any new loss of taste or smell?

5. Any positive test for COVID-19 within the previous 14 days?

6. Any contact with confirmed COVID-19 cases in the last 14 days?

Co-Diagnostics (Salt Lake City, USA) platforms. Each test assessed multiple target genes (a combination of ORF, $\mathrm{N}$, $\mathrm{S}, \mathrm{RdRp}$ ) up to a cycle threshold (Ct) of 40 cycles. Rapid antigen testing was conducted using COVID-19 Ag Rapid Test Device (Abbott Rapid Diagnostics Jena GmbH; Orlaweg 1, 07743 Jena, Germany). In asymptomatic individuals, the rapid antigen testing (lateral flow test) has been reported to have a specificity of $100 \%$ and a sensitivity of $66 \%$ (sensitivity is $94 \%$ with $\mathrm{Ct}$ values $<30$ ). ${ }^{19}$ The RT-PCR testing used is reported to have specificity of $>99 \%$ and sensitivity of $>98 \%{ }^{20}$

Testing, daily symptom and contact checks, and daily temperature checks were tracked through an event accreditation and tracking application (RFID, London, UK) (table 1). Any abnormality was referred to the tournament infection control officer and doctor, who in turn consulted with the public health authority and both conducted contact tracing (figure 1). When a case was identified, a full travel and contact history was taken as well as a risk assessment of the initial contact. Persons considered high-risk or direct contacts (as per the WHO guidelines $)^{21}$ were isolated. Where all protocols had been followed, including the wearing of filtering face piece (FFP2) masks on flights with high-efficiency particulate absorbing filtration; persons within two rows in any direction in an asymptomatic individual were considered moderate-risk contacts. Persons on the same aircraft but not within two rows in any direction were considered lowrisk contacts.

High-risk contacts were usually asked to isolate for 10 days or according to host government advice. ${ }^{22}$ Moderaterisk contacts received education regarding enhanced medical surveillance, had daily rapid antigen testing for 5 days, with RT-PCR day 5 , mandated mask use and access to outside space for work purposes only. Low-risk contacts received rapid antigen testing every 48 hours and RT-PCR testing on day 5 . The close contact testing for those who were asymptomatic is seen in figure 2. COVID-19 officers and social distancing officers educated and engaged the affected person regarding adherence to protocols and non-pharmaceutical interventions (NPIs).

Following the quantitative data collection (using systems within Cignpost Diagnostics), interviews were conducted with the operational leads from testing, 


\section{PGA European Tour COVID-19 Player Pathway}
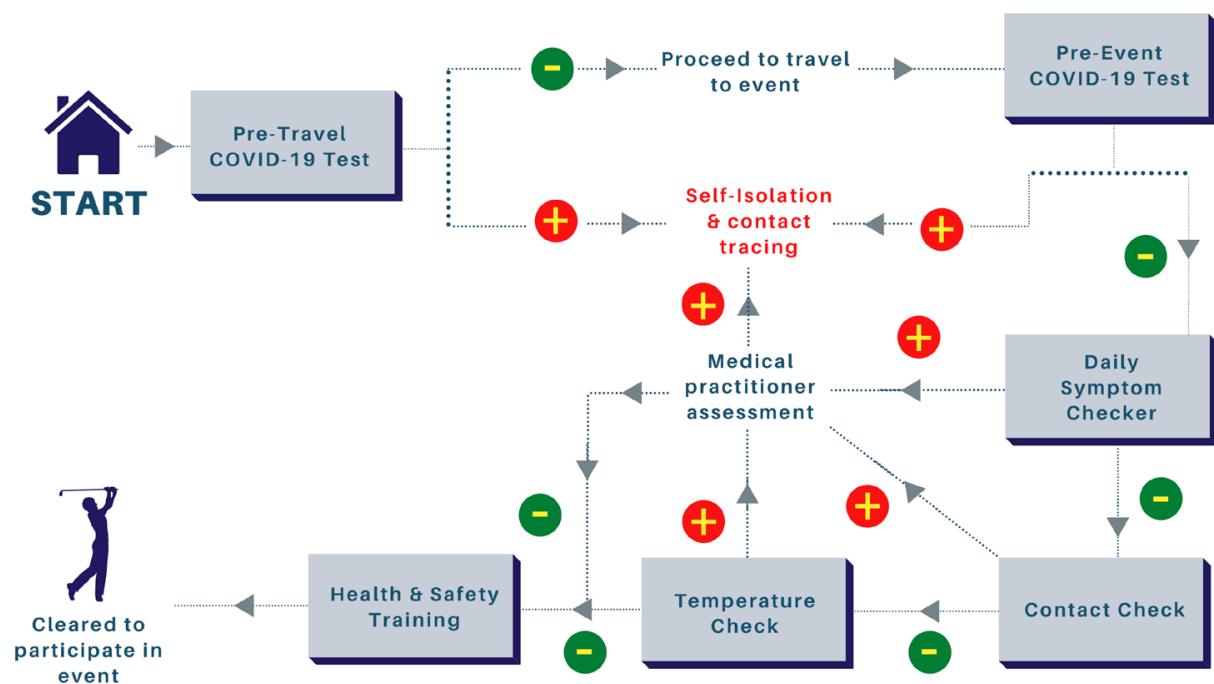

Figure 1 PGA European Tour COVID-19 player pathway.

COVID-19 response, the medical director and the tournament director to understand operational feasibility, behavioural factors and the societal impact of the intervention.

\section{Patient and public involvement}

Patients and/or the public were not involved in the design, or conduct, or reporting, or dissemination plans of this research.

\section{RESULTS}

A total of 550 persons were accredited and required to take PCR tests for the event (age range 18-60 years old). Two of these tests were positive $(0.36 \%)$. Of these, case 1 had one high-risk contact (shared indoor space in a car and within a hotel room for $>15$ min), 23 moderaterisk contacts from two commercial flights (shared flight within two rows in all directions) while 48 were considered

\section{Serial testing approach using lateral flow rapid antigen / polymerase chain reaction testing

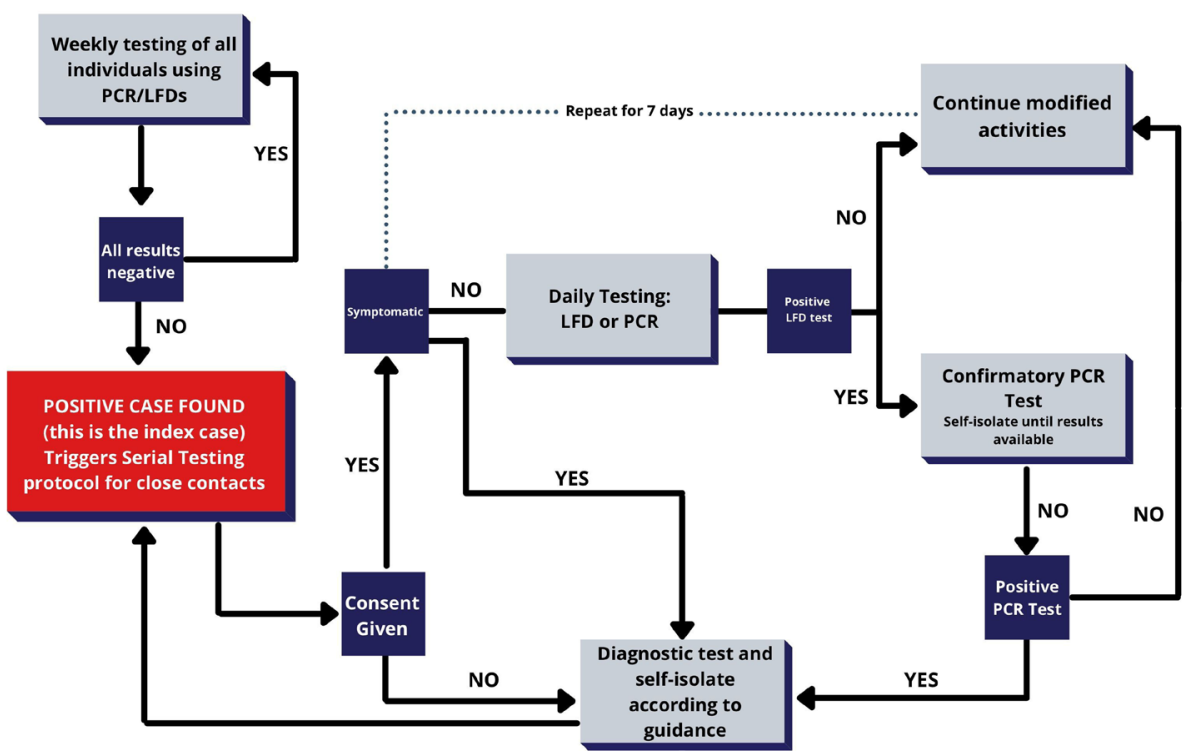

Figure 2 Serial testing approach using lateral flow rapid antigen (LFD)/Polymerase chain reaction (PCR) testing. 
low-risk contacts, having been on the same aeroplane, but out with the two rows in all directions specified by the public health guidance received. Case 1 wore an FFP2 mask throughout the flight and did not move within the cabin and did not have any COVID-19 defining symptoms at the time of the positive test. Case 2 did not have any significant travel history within 2 days of positive test and had one high-risk contact from shared outdoor and indoor space (dining but not room share) within 48 hours. Case 2 was asymptomatic at time of testing and subsequently developed minor symptoms.

Of the two high-risk contacts (one from exposure to case 1 and one from exposure to case 2), both remained negative by RT-PCR on day 10 and were discharged from further investigations. Neither had been vaccinated nor had a previous COVID-19 diagnosis. Of the 23 moderaterisk contacts, all had RT-PCR testing on day 0 and day 5 following exposure and rapid antigen test on day 1 through day 4 . All tests were negative. There were a total of 48 low-risk contact, which received RT-PCR testing on day 0 and day 5 (or 6 ) and rapid antigen testing on day 2 and day 4 . All tests were negative. Attendance for routine and additional testing was $100 \%$.

There were no further positive tests on site in the wider cohort of attendees, which included a total of 872 RT-PCR and 198 rapid antigen tests. One person on site was tested for non-COVID-19 defining symptoms (lethargy), with no persons (other than case 2) presenting with a new continuous cough, shortness of breath, loss of taste or smell or a fever. Persons falling into the category of moderate and low-risk contacts were accepting of the daily testing regime, and the enhanced risk management employed with good compliance. Medical, technical and operational staff did not report difficulty with the workload produced from the enhanced testing and protocols. This increased requirement fell well within the capacity of the on-site laboratory, which is capable of 500 RT-PCR and 500 rapid antigen tests per day if required.

\section{DISCUSSION}

This pilot study has shown that daily testing and minimised risk environment protocols are practical and feasible at a professional golf event with 550 attendees. Initial tests of $0.36 \%$ were positive with no transmission or subsequent positive tests in other contacts regardless of risk. This event was held during high/very high prevalence rates of COVID-19 in Europe, when judged by WHO criteria (426 per 100000 persons) ${ }^{23}$ Both attendees testing positive had arrived from locations of high prevalence (judged by $>350 / 100000$ cases/day) and external to golf tournament 'bubbles'.

A blanket strategy to isolate all known contacts would have caused serious disruption and possible cancellation of the golf tournament.

\section{Operational feasibility}

Elite and professional sport have put in place risk assessment and comprehensive measures aimed at reducing the incidence, prevalence and transmission of COVID$19{ }^{6-1024}$ Key factors to take into account are related to (a) the initial 'contact' exposure and (b) the risk of onward transmission. Similar tiered, risk-mitigating strategies have been implemented in North America across their national professional leagues of football, soccer, hockey, basketball and baseball. ${ }^{25}$ However, their tiers were based on a person's role within an organisation/event, for example, player/team staff versus event staff versus housekeeping. This is in comparison to the present study where risk was categorised on an individual, situational basis with specific consideration given to the circumstances of contacts on transport to the event (eg, air travel) and where the level of enhanced testing for such persons was based on the risk of the contact episode.

This pilot evaluation assessed whether it is feasible and practical to put in place daily testing and enhanced protocols to allow participants to continue to work in a professional sports setting. Participants were compliant with measures attending for $100 \%$ of the required testing and without any significant breach of protocol. The tournament director was clear that the additional testing, although creating extra workload, was worthwhile in ensuring the event could continue. Delivery leads were clear that the work could be accommodated, and a continuation of the programme is practical and feasible. Professional golfers are generally used to following strict rules as part of the sport and have developed a level of trust in providers, having used the same operations and testing team throughout the pandemic. This may or may not be applicable in other settings. Staff and players were aware that the sanction for non-compliance was strict isolation and disqualification from participation. Although not required in this pilot, confirmatory RT-PCR testing following positive rapid antigen testing was immediately available and would have been employed if necessary.

The tournament director expressed his concerns regarding the loss of 75 individuals (2 cases and 73 contacts) out of 550, which would compromise the integrity of the event and in many instances lead to cancellation. This would directly impact the ability of those 550 persons to work and would have a substantial impact on each individual, the host venue and its infrastructure. In addition, such late notice cancellations would be extremely disruptive and have significant ramifications on the professional golf calendar.

\section{Scientific knowledge and implications for policy}

Contact tracing, along with robust testing, isolation and support of cases, is of paramount importance in stopping transmission of SARS-CoV-2. ${ }^{11}$ As sport opens up more to international travel, including the Tokyo Olympic Games, and major events in football, tennis, and motor racing, increased knowledge of factors related to transmission $^{12}$ and measures to decrease risk at events are paramount. ${ }^{26}$ The findings from this present study can inform strategies to both limit disease transmission and 
permit sporting and other events to occur and, therefore, enable the social and economic benefits available.

Accurate risk assessment, protocols to limit risk during travel, testing and vaccination strategies to facilitate events while minimising risk are necessary to allow sport to continue without disruption. ${ }^{27}$ With international travel, and increased attendance at events, the number of contacts sports persons and their essential support personnel may accrue can be large, even if all reasonable precautions are taken. In some regions, there is a legal requirement to self-isolate, ${ }^{28}$ while in other settings, there is consideration for vaccine status, initial contact status, risk and impact of transmission and the measures that can be deployed to reduce transmission risk. ${ }^{29}$ This study did not demonstrate any transmission related to exposure to a case on an aircraft. While this is reassuring, further conclusions cannot be drawn given the low case numbers included. However, this study did demonstrate that protocols regarding risk-mitigated travel can be put in place and can be adhered to. It also demonstrated that enhanced protocols and daily rapid antigen testing are practical, feasible and well accepted in this setting.

Risk minimisation and daily testing may enable persons to be able to continue sporting or work activity, rather than to self-isolate. Public health rationale is additionally based on modelling, which shows daily testing without quarantine after tracing may avert a similar proportion of onward disease transmission from secondary cases compared with that of a 14-day quarantine $(50 \%, 95 \%$ UI 23-81; RR 0.88, 0.60-1.43). ${ }^{30}$ This modelling was based on at least 5 days of daily testing. A risk assessment and enhanced testing may be appropriate if the following conditions are met:

- Agreement is reached with the public health authority and event organiser.

- Risk is assessed.

- Control measures can return risk to a baseline or acceptable level.

- Daily/regular testing is immediately available.

- Medical oversight is strong and immediately available. Further pilot work at golf and other professional sporting events could inform a strategy by which when a positive case is detected in an international sporting setting, risk is assessed, risk mitigations including NPIs are enhanced, and close contacts are tested daily using rapid antigen testing instead of self-isolation of individuals who test negative. If a person has a negative rapid antigen test within 24 hours, this will then provide a 'passport' for that person to undertake their normal or modified daily activities. It is reassuring that an event based in a region of high daily rates of COVID-19 had positive cases in only $0.36 \%$ of the attendees. However, we recognise that this is not absolutely comparable to local rates given our entire study population was tested.

This proposed process is reliant on the ability to obtain a rapid test result, such as what has been possible in this current study. Antigen lateral flow devices currently give the quickest result turnaround of all the COVID-19 tests with results typically available in under an hour. RT-PCR tests have a greater sensitivity than rapid antigen testing ${ }^{31}$ and could also be used in this setting with the turnaround time for RT-PCR $<4$ hours in $90 \%$ of cases where on site labs are available on European Tour. The optimal interval between tests is still being evaluated, but it is anticipated that daily testing will occur until such time as this needs to change in the light of experience. If a person tests positive during this process, they will then be required to undertake a confirmatory RT-PCR test and self-isolate for 10 days from the date of the positive test result in the normal way. In addition, risk mitigation efforts may require to be heightened or relaxed depending on evolving features of the pandemic such as new virus variants or an increase in double-vaccinated persons. Clinicians designing riskmitigating processes must be flexible to change as factors of the pandemic progress such as new variants escaping current vaccine protection.

\section{CONCLUSION}

Accurate risk assessment, implementation of riskmitigating measures, testing, contact tracing and support of cases and contacts are of paramount importance in stopping transmission of SARS-CoV-2. This pilot study showed it is practical, feasible and well accepted to provide enhanced (daily) virus testing and risk-mitigating measures at a professional golf event. Further study is required to assess the efficacy of these interventions. However, no transmission was found in this pilot study with these enhanced measures. These interventions are reliant on suitably trained staff, and a cohort of participants willing to undertake daily testing. The protocols established in this study have led to a pilot multi-sport approach in countries such as England, where the research is being taken forward in collaboration with the Department of Health and Social Care. It may inform further study and implementation regarding contact tracing for elite sport.

\section{Twitter Andrew Murray @docandrewmurray}

Acknowledgements Colleagues at the World Health Organisation, and various international governing bodies for sport (ATP, FIFA, F1, the IOC, PGA Tour and World Rugby) were instrumental in shaping risk assessment and risk mitigation policies.

Contributors PGR—data analysis, manuscript writing, final approval of manuscript. AM — research idea, data analysis, manuscript writing, final approval of manuscript. GC-manuscript writing, final approval of manuscript. VSmanuscript writing, final approval of manuscript. DFK— manuscript writing, final approval of manuscript.

Funding The authors have not declared a specific grant for this research from any funding agency in the public, commercial or not-for-profit sectors.

Competing interests AM and GC have paid roles with The R\&A, the European Tour and The Ladies European Tour. VS has a paid role with the European Tour. DFK is the Founder and Chief Medical Officer of Cignpost Diagnostics Ltd.

\section{Patient consent for publication Not required.}

Ethics approval Ethical approval was granted by the local ethics committee of Liverpool John Moores University (21/SPS/027).

Provenance and peer review Not commissioned; externally peer reviewed.

Data availability statement Data are available upon reasonable request. The data can be accessed on request of the senior author. 
Open access This is an open access article distributed in accordance with the Creative Commons Attribution Non Commercial (CC BY-NC 4.0) license, which permits others to distribute, remix, adapt, build upon this work non-commercially, and license their derivative works on different terms, provided the original work is properly cited, appropriate credit is given, any changes made indicated, and the use is non-commercial. See: http://creativecommons.org/licenses/by-nc/4.0/.

ORCID iD

Patrick G Robinson http://orcid.org/0000-0002-8117-2968

\section{REFERENCES}

1 Lee I-M, Shiroma EJ, Lobelo F, et al. Effect of physical inactivity on major non-communicable diseases worldwide: an analysis of burden of disease and life expectancy. Lancet 2012;380:219-29.

2 Chastin SFM, Abaraogu U, Bourgois JG, et al. Effects of regular physical activity on the immune system, vaccination and risk of community-acquired infectious disease in the general population: systematic review and meta-analysis. Sports Med 2021;51:1673-86.

3 World Health Organisation. Key planning recommendations for mass Gatherings in the context of COVID-19, 2021. Available: https:// www.who.int/publications-detail/key-planning-recommendationsfor-mass-gatherings-in-the-context-of-the-current-covid-19outbreak

4 World Health Organisation. Considerations for sports federations/ sports event organizers when planning mass gatherings in the context of COVID-19, 2020. Available: https://apps.who.int/ iris/bitstream/handle/10665/331764/WHO-2019-nCoV-Mass Gatherings Sports-2020.1-eng.pdf

5 World Health Organisation. Mass gathering sporting risk assessment, 2021. Available: https://www.who.int/emergencies/ diseases/novel-coronavirus-2019/technical-guidance/points-ofentry-and-mass-gatherings

6 Kemp S, Cowie CM, Gillett M, et al. Sports medicine leaders working with government and public health to plan a 'return-to-sport' during the COVID-19 pandemic: the UK's collaborative five-stage model for elite sport. Br J Sports Med 2021;55:4-5.

7 Hughes D, Saw R, Perera NKP, et al. The Australian Institute of sport framework for rebooting sport in a COVID-19 environment. J Sci Med Sport 2020;23:639-63.

8 Robinson PG, Murray A, Close G, et al. Assessing the risk of SARSCoV-2 transmission in international professional golf. BMJ Open Sport Exerc Med 2021;7:e001109.

9 Jones B, Phillips G, Kemp S, et al. SARS-CoV-2 transmission during rugby League matches: do players become infected after participating with SARS-CoV-2 positive players? Br J Sports Med 2021;55:bjsports-2020-103714.

10 Schumacher YO, Tabben M, Hassoun K, et al. Resuming professional football (soccer) during the COVID-19 pandemic in a country with high infection rates: a prospective cohort study. $\mathrm{Br} \mathrm{J}$ Sports Med 2021. doi:10.1136/bjsports-2020-103724. [Epub ahead of print: 15 Feb 2021].

11 World Health Organisation. Surveillance, case investigation and epidemiological protocols: contact tracing in the context of COVID-19, 2021. Available: https://www.who.int/publications/i/item/ contact-tracing-in-the-context-of-covid-19

12 Koh WC, Naing L, Chaw L, et al. What do we know about SARSCoV-2 transmission? A systematic review and meta-analysis of the secondary attack rate and associated risk factors. PLoS One 2020;15:e0240205.

13 World Health Organisation. COVID-19 strategy update, 2021. Available: https://www.who.int/publications/m/item/covid-19strategyupdate
14 World Health Organisation. Critical preparedness, readiness and response actions for COVID-19 (interim guidance), 2021. Available: https://www.who.int/publications-detail/critical-preparednessreadiness-and-response-actions-for-covid-19

15 Chu DK, Akl EA, Duda S, et al. Physical distancing, face masks, and eye protection to prevent person-to-person transmission of SARS-CoV-2 and COVID-19: a systematic review and meta-analysis. Lancet 2020;395:1973-87.

16 Public Health Ontario. Risk assessment approach for COVID-19 contact tracing, 2021. Available: https://www.publichealthontario. ca/-/media/documents/ncov/main/2020/09/covid-19-contacttracing-risk-assessment.pdf?la=en

17 Perrault A, Charpignon M, Gruber J. Designing efficient contact tracing through Risk-Based Quarantining. medRxiv 2020.

18 Rankin A, Massey A, Falvey Éanna Cian, et al. Infographic. COVID-19 RT-PCR testing for elite athletes. Br J Sports Med 2021;55:818-20.

19 Abbott Rapid Diagnostics Jena $\mathrm{GmbH}$. In vitro diagnostic rapid test for qualitative detection of SARS-CoV-2 antigen (Ag), 2021.

20 Cignpost Diagnostics. About Cignpost diagnostics, 2021. Available: https://www.cignpostdiagnostics.com/about-cignpost [Accessed 26 Jun 21].

21 World Health Organisation. Contact tracing in the context of COVID-19 2020. Available: https://www.who.int/publications/i/ item/contact-tracing-in-the-context-of-covid-19 [Accessed 21 May 21].

22 Publich Health England. Stay at home: guidance for households with possible or confirmed coronavirus (COVID-19) infection: Gov.uk, 2021. Available: https://www.gov.uk/government/publications/covid19-stay-at-home-guidance/stay-at-home-guidance-for-householdswith-possible-coronavirus-covid-19-infection

23 European Centre for Disease Transmission and Control. Weekly surveillance summary. COVID-19 country Overviews 2021. Available: file://Users/patrickrobinson/Downloads/country_overview_report_ 20210422\%203.html

24 Meyer T, Mack D, Donde K, et al. Successful return to professional men's football (soccer) competition after the COVID-19 shutdown: a cohort study in the German Bundesliga. Br J Sports Med 2021;55:62-6.

25 DiFiori JP, Green G, Meeuwisse W, et al. Return to sport for North American professional sport leagues in the context of COVID-19. Br J Sports Med 2021;55:417-21.

26 Carmody S, Murray A, Borodina M, et al. When can professional sport recommence safely during the COVID-19 pandemic? risk assessment and factors to consider. $\mathrm{Br} J$ Sports Med 2020;54:946-8.

27 Murakami M, Yasutaka T, Onishi M. Living with COVID-19: mass gatherings and minimizing risk. Qjm 2021;372.

28 Australian Government Department of Health. Coronavirus (COVID-19) advice for international travellers [16/6/21]. Available: https://www.health.gov.au/news/health-alerts/novel-coronavirus2019-ncov-health-alert/coronavirus-covid-19-restrictions/ coronavirus-covid-19-advice-for-international-travellers

29 Foreign and Commonwealth Office. Greece. entry rules in response to coronavirus (COVID-19). Available: https://www.gov.uk/foreigntravel-advice/greece/entry-requirements

30 Quilty BJ, Clifford S, Hellewell J, et al. Quarantine and testing strategies in contact tracing for SARS-CoV-2: a modelling study. Lancet Public Health 2021;6:e175-83.

31 Porton Down PHE UoO. Preliminary report: rapid evaluation of latera flow viral antigen detection devices (LFDs) for mass community testing, 2020. Available: https://www.ox.ac.uk/sites/files/oxford/ media_wysiwyg/UKevaluation_PHEPortonDownUniversityofOxford_ final.pdf 